\title{
Infective Spondylitis - A Complication of Temporary Catheter inPatient Receiving Haemodialysis: A Case Report
}

\author{
HAQUE WMM ${ }^{\mathrm{a}}$, SAMAD T ${ }^{\mathrm{b}}$, ANANNA MA ${ }^{\mathrm{c}}$, RAHIM MA ${ }^{\mathrm{d}}$, IQBAL $\mathrm{S}^{\mathrm{e}}$
}

\begin{abstract}
:
Infective spondylodiscitis (ISD) is an uncommon, devastating but potentially preventable and treatable condition specially in patients receiving haemodialysis (HD) through central venous (CV) catheters. Here, we present an elderly diabetic patient who was on HD through CV line placed in right femoral vein, who came because of pyrexia
\end{abstract}

\section{Introduction:}

Intravascular catheter-related infections are major causes of morbidity and mortality. Common aetiological agents include coagulase-negative Staphylococci, Staphylococcus aureus, aerobic Gramnegative bacilli, and Candida albicans. ${ }^{1}$ Management of catheter-related infection varies according to the type of catheter involved. Temporary haemodialysis catheters have a high failure rate associated with a significant rate of complications. Infective spondylodiscitis (ISD) is a rare but devastating condition in hemodialysis (HD) patients. The diagnosis of pyogenic spinal infection should be considered in any patient presenting with localized, unremitting back or neck pain, especially when accompanied with

a. Dr. Wasim Md. MohosinulHaque, FCPS(Medicine), Associate Professor, Nephrology, Ibrahim Medical College and BIRDEM general Hospital, Shahbag, Dhaka 1000, Bangladesh

b. Dr. TabassumSamad, FCPS(Medicine), Registrar, Nephrology, Ibrahim Medical College and BIRDEM general Hospital, Shahbag, Dhaka 1000, Bangladesh

c. Dr. MehrubaAlamAnanna, FCPS(Medicine), Asisstant Professor, Nephrology, Ibrahim Medical College and BIRDEM general Hospital, Shahbag, Dhaka 1000, Bangladesh

d. Dr. Muhammad Abdur Rahim, FCPS(Medicine), Junior Consultant, Nephrology, Ibrahim Medical College and BIRDEM general Hospital, Shahbag, Dhaka 1000, Bangladesh

e. Dr. SarwarIqbal,MD(Nephrology), Associate Professor, Nephrology, Ibrahim Medical College and BIRDEM general Hospital, Shahbag, Dhaka 1000, Bangladesh

Address of correspondance: Dr. TabassumSamad, FCPS (Medicine), Registrar, Nephrology, Ibrahim Medical College and BIRDEM general Hospital, Shahbag, Dhaka 1000, Bangladesh. Email: samadtabassum@yahoo.com.

Receive: 23 July 2013

Accecpted: 10 February 2014 and neck pain. Diagnostic workup was in favour of ISD and excluded tuberculosis or malignancy. He responded well to anti-staphylococcal antibiotics.

Key words: central venous catheters, infective spondylodiscitis, haemodialysis.

(Birdem Med J 2014; 4(2):108-110)

systemic features and in presence of increased inflammatory markers. More emphasis should be given on ISD, if a patient on HD through central venous (CV) line. Here, we present a case of non-tubercular spondylitis in a patient on HD due to intravascular catheter related infection.

\section{Case Report:}

A 62-year-old diagnosed case of diabetes mellitus (DM), hypertension end stage renal disease (ESRD) on MHD through CV line presented with 2 week history of continued fever reaching up to $104^{\circ} \mathrm{F}$ and neck pain. The neck pain was constant, dull aching with radiation to upper limbs and aggravated on movement. There was no history of trauma. His dialysis first started through right femoral vein catheter 1 month back and continued until it was changed by another one in the same site because of development of shivering during HD. His fever started a week after the reinsertion of the CV line. There were no other focal symptoms suggestive of fever origin.

On admission, his temp was $102^{\circ} \mathrm{F}$. He was anaemic and oedematous. His pulse was $96 / \mathrm{min}$ and blood pressure 170/100 mm Hg. ACV line was in situ at right femoral vein, exit site was healthy. There was grade III tenderness over $\mathrm{C}_{6}$ vertebra and restriction of cervical spinal movement in all direction. Chest, precordium and abdominal examination did not reveal any abnormality. He had diabetic retinopathy.

After sending culture samples, we removed the CV line and his HD was continued through already matured arterio-venous fistula. His antibiotic was changed from ceftazidime to vancomycin. 
His $\mathrm{Hb}$ was $9.2 \mathrm{gm} / \mathrm{dl}$, normochromic, normocytic, total and differential WBC and platelet count was normal, ESR $75 \mathrm{~mm}$ in $1^{\text {st }}$ hour and CRP $10 \mathrm{mg} / \mathrm{dl}$. Blood, catheter tip and urine cultures showed no growth. X-ray cervical spine revealed degenerative change and reduced joint space between $\mathrm{C}_{5}-\mathrm{C}_{6}$ (Figure 1). MRI findings of cervical spine were suggestive of infective/ inflammatory discitis $\left(\mathrm{C}_{5}-\mathrm{C}_{6}\right)$ (Figure 2). CT guided FNAC (Figure 3 ) showed only few polymorphs, lymphocytes and histiocytes which were compatible with infective cause and no evidence of granuloma or malignancy was found. Chest $\mathrm{X}$-ray, Monteux test, brucella serology were normal. Abdominal ultrasonography showed renal parenchymal disease. His predialysis serum creatinine was $9.8 \mathrm{mg} / \mathrm{dl}$, serum electrolytes were normal. His fasting blood glucose was $7.3 \mathrm{mmol} / \mathrm{l}$.

So, the patient was diagnosed as a case of DM,

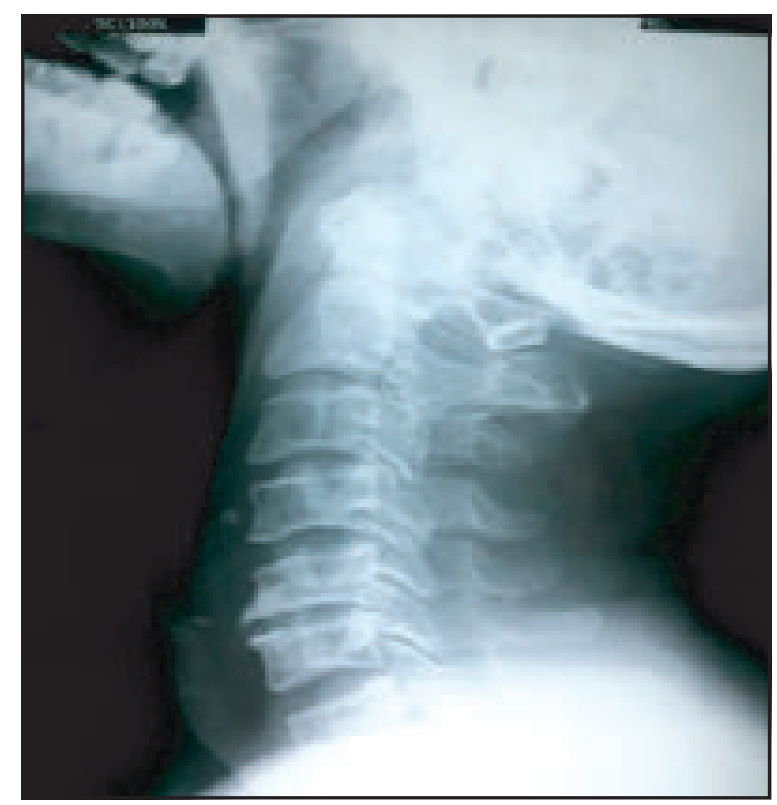

Fig 1: $X$-ray cervical spine (lateral view) showing redued space in $C_{5}-C_{6}$.

hypertension, ESRD on MHD and ISD. His fever subsided with vancomycin and he was discharged with oral flucloxacillin and ciprofloxacin and spinal bracing. On follow up visit after 4 weeks he further improved (Figure 4). We could not follow up the patient with repeat MRI.

\section{Discussion:}

\section{9}

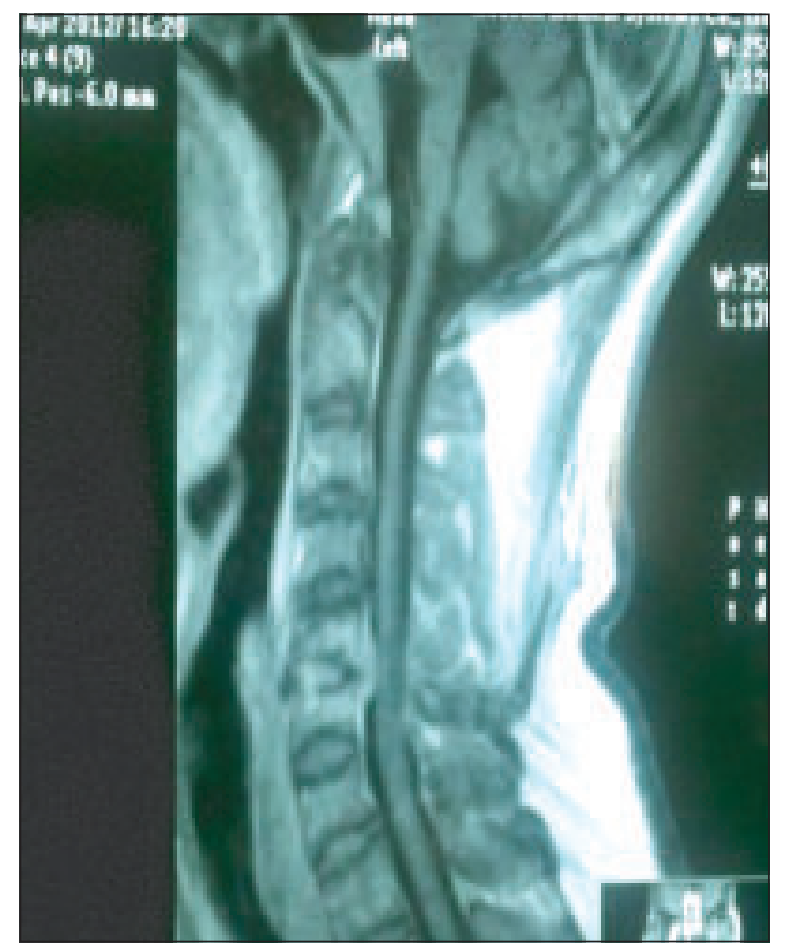

Fig 2: MRI cervical spine showing infective / inflammatory discitis $\left(C_{5}-C_{6}\right)$

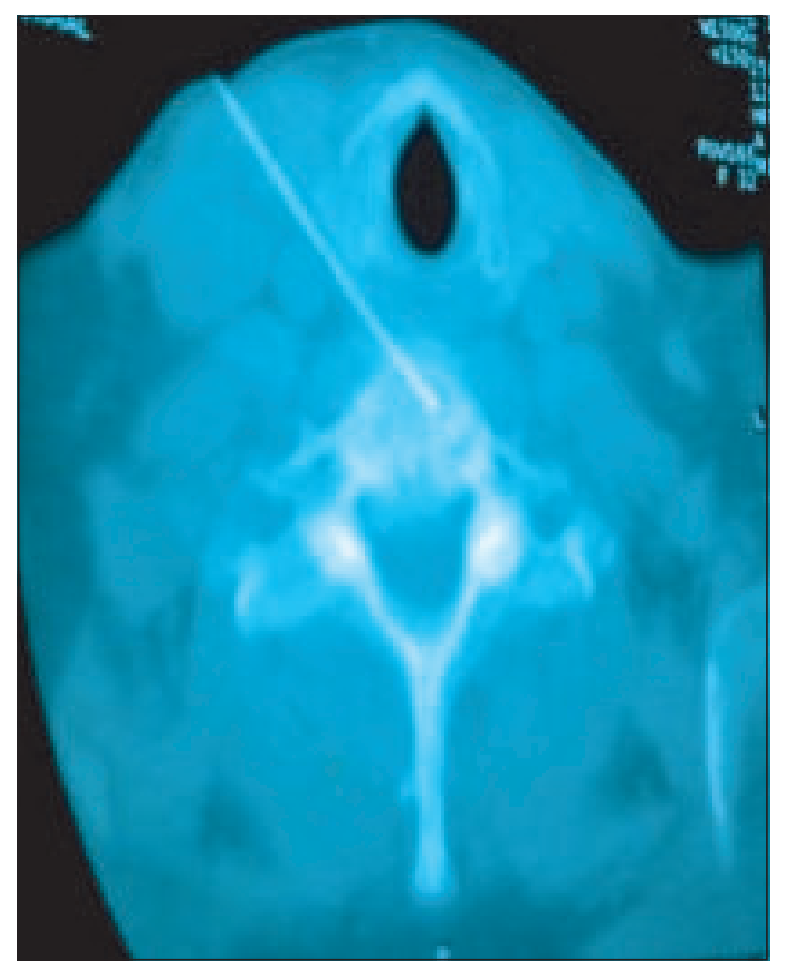

Fig 3: CT guided FNAC from cervical vertebra 


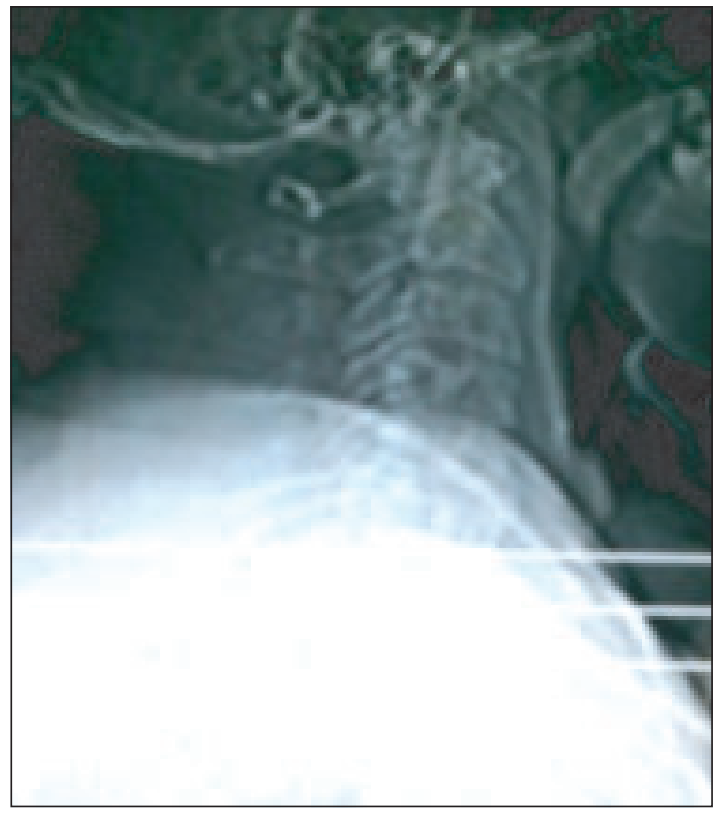

Fig 4: X-ray cervical spine during follow up

Although bacterial spondylodiscitis is one of the most serious complications in hemodialysis patients, it is often under-diagnosed and few reports describe its course and management. In a retrospective study it was found that ISD occurred mostly in elderly male patients and thoracic spine $(53 \%)$ was most commonly affected site followed by lumber (33\%) and cervical (7\%) spine. ${ }^{2}$ Our patient was male and elderly but the involved site was cervical spine.

Fever is the commonest presentation and CV line specially multiple vascular access is an important risk factor. ${ }^{3}$ Other common risk factors included DM (62\%), malnourishment (54\%), cirrhosis (31\%), ESRD (15\%), intravenous drug use (15\%) and multiple risk factors $(76 \%)^{2}$ The main port of bacterial entry were intravenous catheter $(23 \%)$, urinary tract $(15 \%)$ and intravenous drug use $(15 \%){ }^{2}$ Use of femoral venous catheter is an independent risk factor ${ }^{4}$ and placement in an old site is another risk factor for blood stream infection. ${ }^{5}$ Our patient was diabetic and ESRD on MHD through femoral line.

Staphylococcus aureus is the commonest organism. ${ }^{3,6} \mathrm{In}$ our patient, we could not isolate any organism possibly as he was on antibiotic for a long period. We treated him with vancomycin followed by flucloxacillin and ciprofloxacin. In a retrospective study, no death occurred in patients treated with vancomycin and gentamycin. ${ }^{3}$ Conservative measures including prolonged antibiotic therapy and spinal bracing was proved successful in many cases. ${ }^{6}$ We also treated our patient with antibiotics and adjusted the duration of antibiotic therapy depending on clinical and laboratory evidence until the patient was improved but we could not monitor him by MR gadolinium scan.

\section{Conclusion:}

ISD must be suspected whenever a patient on hemodialysis present with fever and/ or back pain. The presence of a CV catheter should increase the threshold for suspicion. Prolonged antibiotic therapy with initial broad-spectrum coverage seems to be the best therapeutic approach. Preventive strategies to reduce the prevalence of bacteremia, including sterility during $\mathrm{CV}$ line insertion and its care should be an overriding aim.

\section{References:}

1. Mermel LA, Farr BM, Sherertz RJ, Raad ii, O’gardy N, Harris JS, et al. Guidelines for the management of intravenous catheter related infection. J IntravenNurs 2001; 24(3):180-205.

2. Al-Nammari SS, Lucas JD, Lam KS. Hematogenous methicillin-resistant Staphylococcus aureusspondylodiscitis. Spine $2007 ; 32(22): 2480-86$.

3. Faria B, Canto Moreira N, Sousa TC, Pêgo C, Vidinha J, Garrido J, et al. Spondylodiscitis in hemodialysis patients: a case series. ClinNephrol2011; 76(5):380-87.

4. Ishizuka M, Nagata H, Takagi K, Kubota K. Femoral venous catheterization is a major risk factor for central venous catheter related blood stream infection. J Invest Surg 2009;22(1):16-21.

5. Safdar M, Kluger DM, Maki DG. A review of risk factor for central venous catheter related blood stream infection caused by percutaneously inserted non cuffed central venous catheter: implication for preventive stratigies .Medicine (Baltimore) $2002 ; 81(6): 466-79$.

6. Bettini N, Girardo M, Dema E, Cervellati S. Evaluation of conservative treatment of nonspecific spondylodiscitis. Eur Spine J 2009 ;18 (Suppl 1):143-50. 